\title{
Effects of Tube Diameter and Length on Transparent Conductivity of Single-Walled Carbon Nanotube Network Films
}

\author{
Yuki Kuwahara $\mathbb{D}^{1,2}$ Takayoshi Hirai, $^{2}$ and Takeshi Saito $\mathbb{D D}^{1,2}$ \\ ${ }^{1}$ Nanomaterials Research Institute, National Institute of Advanced Industrial Science and Technology (AIST), 1-1-1 Higashi, \\ Tsukuba, Ibaraki 305-8565, Japan \\ ${ }^{2}$ Technology Research Association for Single Wall Carbon Nanotubes (TASC), 1-1-1 Higashi, Tsukuba, Ibaraki 305-8565, Japan \\ Correspondence should be addressed to Yuki Kuwahara; yuki.asada@aist.go.jp and Takeshi Saito; takeshi-saito@aist.go.jp
}

Received 12 April 2018; Revised 3 September 2018; Accepted 18 September 2018; Published 25 October 2018

Academic Editor: Miguel A. Correa-Duarte

Copyright $\odot 2018$ Yuki Kuwahara et al. This is an open access article distributed under the Creative Commons Attribution License, which permits unrestricted use, distribution, and reproduction in any medium, provided the original work is properly cited.

The effects of single-walled carbon nanotube (SWCNT) structures on the transparent conductivity of their network films have been investigated. SWCNTs with different average tube diameters of $1.3 \mathrm{~nm}, 1.7 \mathrm{~nm}$, and $2.0 \mathrm{~nm}$ were processed at the same conditions. Then unit structure characterization was performed by determining the tube diameter, length, bundle thickness, and so on, before the fabrication of SWCNT network films by the filtration and transfer method using the dispersions. The result of the transparent conductivity measurements clearly showed better performance with a decrease in the tube diameter: that is, narrower SWCNTs form narrow bundles, and their dense network results in an increase in the total length of conduction pathways in the SWCNT network films with high transparency. Furthermore, the figure of merit and the percolation exponent for the transparent conductivity obtained by using the data fitting of the percolation model were also discussed in terms of the tube diameter and length.

\section{Introduction}

The single-walled carbon nanotube (SWCNT) network film has attracted considerable attention as one of the emerging conductive materials for transparent conductive film (TCF) applications [1]. Because SWCNTs can be dispersed not only in organic solvents [2] but also in aqueous solvents with the help of surfactants [3] or polymers [4], the printing process using the dispersion as the ink is applicable for the preparation of SWCNT network films. Thus, easy scale-up of the film production at a low cost by using existing technology on various substrates $[5,6]$ is the advantage of SWCNTs as a TCF material. Furthermore, SWCNT network films possess excellent transparent conductivity properties in addition to mechanical strength and chemical stability [7-9]. There have been efforts to improve the transparent conductivity properties of SWCNT network films in order to achieve higher transparency and lower sheet resistance nearly up to the currently demanded levels, achieved via the commonly used acid treatment doping method for the reduction of sheet resistance $[7,8]$. Although maintaining long-term stability of the properties after acid treatment has remained an important issue to be solved, it has recently been reported that photonic curing of a vacuum-evaporated copper halide film on a SWCNT network builds efficient and stable nanotubenanotube interconnects that result in prospective long-term stability [9]. Because of these advances, SWCNTs are one of the promising alternative to current materials, including rare metals, in a variety of applications.

Various structural parameters of the SWCNT network films, such as tube length, tube diameter, bundle thickness, and network connectivity, strongly influence the electronic properties [10-13]. Therefore, clarification of the structural effects on the transparent conductivity of SWCNT network films would be valuable for improving the film fabrication and postprocessing methods. Furthermore, the underlying relationships between structural parameters of the SWCNT network and transparent conductivity are scientifically interesting in terms of the physics associated with complex systems composed of one-dimensional elements [14-16]. Longer tube lengths have been reported to result in higher DC (electrical) conductivity of SWCNT network films [17-19]. Films of 
SWCNTs with metallic chirality also exhibit a high conductivity $[20,21]$. In addition, the performance of SWCNT network films is affected by the method of SWCNT production because the synthesis conditions cause changes in structural characteristics such as purity and crystallinity [22-24].

The tube diameter is one of the important structural parameters that have a profound influence over the properties of SWCNT network films because of the considerable correlation between the tube diameter and the electronic structure and carbon atom density [25]. In a recent work on the effects of the bundle thickness on SWCNT network films [26], a preliminary study on the effects of the tube diameter was also conducted. However, a correlation between the transparent conductivity and tube diameter was not observed, probably because of the broad tube diameter distribution. For clarifying the effect of the tube diameter on the properties of SWCNT network films, achieving homogeneity of each structural feature is necessary. Therefore, despite many studies on structural effects on the transparent conductivity of SWCNT network films, scope for further research exists.

In this study, we have investigated the effects of structural parameters, especially the tube diameter, on the transparent conductivity properties of SWCNT network films prepared by the filtration and transfer method [1], in which the tube length and diameter of SWCNTs were independently varied. The results showed that the transparent conductivity of SWCNT network films is definitely influenced by the tube diameter that affects the network density. In addition, the transparent conductivity has been also analyzed based on the percolation theory [27].

\section{Experimental Methods}

Groups of SWCNTs with different tube diameters were synthesized by gas-phase chemical vapor deposition (CVD) growth using the enhanced direct injection pyrolytic synthesis (eDIPS) method [28] and were characterized by UV-visNIR absorption spectroscopy (U-4100, Hitachi High-Tech) and resonance Raman scattering (NRS-5100, Jasco) to determine their tube diameter distributions [29, 30]. $1 \mathrm{mg}$ of SWCNTs was dispersed in $10 \mathrm{ml}$ of aqueous solution of $1 \mathrm{wt} \%$ sodium cholate (SC) by using a probe-type sonicator (Sonifier 450D, Branson) with an output power of $5 \mathrm{~W}$, for $10 \mathrm{~min}$. SC provided sufficient dispersibility irrespective of the SWCNT tube diameter. The dispersions were then centrifuged at $100,000 \times \mathrm{g}$ for $10 \mathrm{~min}$ (UC10) or $60 \mathrm{~min}$ (UC60), and the supernatants of the upper $50 \%$ were applied for the film fabrication. In addition, after centrifugation, the resulting dispersions were characterized by UV-vis-NIR spectroscopy to determine the SWCNT concentration. The lengths of SWCNTs in the dispersions were also characterized by atomic force microscopy (AFM; Dimension Icon, Bruker AXS K.K.). For the preparation of the specimens for AFM, we deposited the dispersions on Si wafers, washed with Milli-Q water, and then dried with blowing air.

In this work, SWCNT network films were fabricated by the filtration and transfer method as previously reported [1]. The as-prepared dispersions were diluted with Milli-Q water to below the critical micelle concentration (ca. $0.6 \mathrm{wt} \%)$ right before film fabrication. The effect of the dilution process will be discussed in Section 3.2. The film fabrication was conducted as follows. $25-400 \mu \mathrm{l}$ of the SWCNT dispersion was added into $200 \mathrm{ml}$ of Milli-Q water and stirred at $500 \mathrm{rpm}$ for $5 \mathrm{~min}$. Then the diluted dispersion was filtrated through the membrane (MF-Millipore, filter diameter: $47 \mathrm{~mm}$, pore size: $0.22 \mu \mathrm{m}$ ), and this was followed by the gentle washing of the SWCNT network film on the filter with $200 \mathrm{ml}$ of Milli-Q water to remove SC. The film thickness (and thus the transparency of the SWCNT network film) was adjusted by the loaded amount of the SWCNT dispersion [31]. After drying, the SWCNT network film was transferred on a glass substrate (microscope cover glass, Matsunami Glass Ind. Ltd.) by dissolving the filter with plenty of acetone. The resulting SWCNT network film on the glass substrate was immersed in acetone for $\sim 30 \mathrm{~min}$, and the acetone was changed three times. The SWCNT film was $40 \mathrm{~mm}$ in diameter. The morphology of the SWCNT network film and the bundle thickness were characterized by AFM observations.

We evaluated the transparent conductivity properties, namely, sheet resistance and total light transmittance (specified in ISO 13468), by using a four-probe resistivity meter (Loresta GP MCP-T610, PSP probe, Mitsubishi Chemical Analytech Co. Ltd.) and a haze meter (NDH 4000, Nippon Denshoku Industries Co. Ltd.), respectively. Here, the total light transmittance, which is one of the standard performance indicators, is the transmittance of the visible light with a wavelength range of 380 to $780 \mathrm{~nm}$. (Details are specified in ISO 13468 and JIS K 7361.)

\section{Results and Discussion}

3.1. Tube Diameter and Length of Unbundled SWCNTs. Figure 1(a) shows optical absorption spectra of the SWCNT dispersions with different average tube diameters. The observed peaks that can be assigned to interband transitions of SWCNTs indicate discrete fine structures with a decrease in the tube diameter, probably because the SWCNTs with smaller tube diameters include a limited number of chiral species. By using the relationship between the peak position and the average tube diameter [30], tube diameters of these SWCNT samples were characterized to be ca. $1.3 \mathrm{~nm}$, $1.7 \mathrm{~nm}$, and $2.0 \mathrm{~nm}$. The analysis of radial breathing modes (RBM) observed in the resonance Raman scattering shown in Figure 1(b) validates these average tube diameters.

As previously reported [32], the tube-length distribution range can be controlled by ultracentrifugation of the SWCNT dispersion. In this work, we have adopted 10 and $60 \mathrm{~min}$ of ultracentrifugation times, UC10 and UC60, in order to prepare two kinds of SWCNT dispersions with different average tube lengths. The average tube length and distributions of UC10 and UC60 were characterized by AFM observation as listed in Table 1. (Typical images and length histograms are shown in Figure S1 in Supplementary Materials.) The height profile analysis for AFM images confirms that most SWCNTs are unbundled. Therefore, we assumed that the characterized length is not for SWCNT bundles but is for 


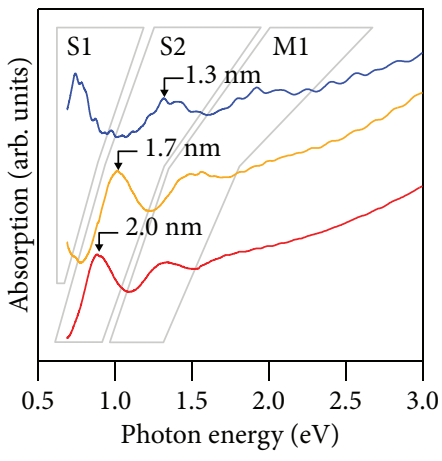

(a)

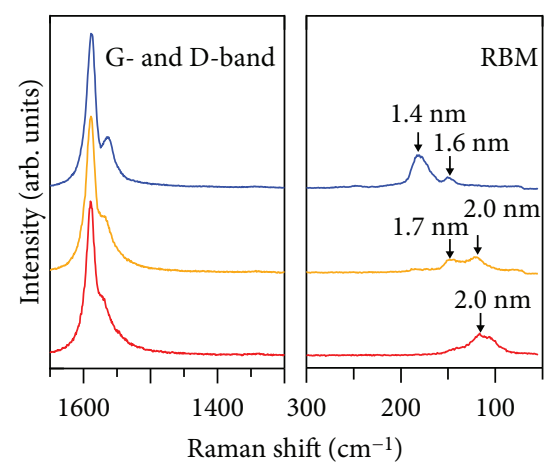

(b)

FIGURE 1: Spectra of UV-vis-NIR absorption (a) and resonance Raman scattering spectra (b) of SWCNTs with different tube diameters. The characterized tube diameters based on the interband transition peaks and RBMs are noted beside the peaks.

TABLE 1: Average tube lengths, bundle thicknesses, and their distributions for SWCNTs characterized by AFM.

\begin{tabular}{|c|c|c|c|c|c|c|}
\hline \multirow{2}{*}{$\begin{array}{l}\text { Tube diameter } \\
\text { Ultracentrifugation time }\end{array}$} & \multicolumn{2}{|c|}{$1.3 \mathrm{~nm}$} & \multicolumn{2}{|c|}{$1.7 \mathrm{~nm}$} & \multicolumn{2}{|c|}{$2.0 \mathrm{~nm}$} \\
\hline & UC10 & UC60 & UC10 & UC60 & UC10 & UC60 \\
\hline Average tube length (nm) & 689 & 634 & 720 & 645 & 530 & 470 \\
\hline Length distribution $( \pm 1 \sigma \mathrm{nm})$ & \pm 485 & \pm 446 & \pm 656 & \pm 425 & \pm 446 & \pm 368 \\
\hline Average bundle thickness (nm) & 5.0 & 4.7 & 5.9 & 5.4 & 9.2 & 8.0 \\
\hline Bundle thickness distribution $( \pm 1 \sigma \mathrm{nm})$ & \pm 2.5 & \pm 2.6 & \pm 3.1 & \pm 2.7 & \pm 4.8 & \pm 4.3 \\
\hline
\end{tabular}

isolated SWCNTs. Table 1 shows that the average tube length of UC10 is surely longer than that of UC60 with the same tube diameter, although the difference between these is moderate. Thus, it was concluded that the longer SWCNTs tend to precipitate a little earlier during ultracentrifugation.

Here, in order to confirm the conservation of the tube diameter distributions during ultracentrifugation, the SWCNT dispersions of UC10 and UC60 were also characterized by optical absorption spectroscopy. Figures 2(a)-2(c) show the gradual precipitation of SWCNTs and the decrease in their concentration in the dispersion during ultracentrifugation. However, no difference in the spectrum shape is observed for each tube diameter, which shows that the tube length is independently affected by ultracentrifugation.

3.2. Morphology of the SWCNT Network Film and Bundle Thickness. SWCNT network films with various transparencies were fabricated by controlling the loaded amounts of the SWCNT dispersions. Figure 3 clearly shows the correlations between the loaded amount of SWCNT dispersions and the total light transmittances (TT) of SWCNT network films, which follow the Lambert-Beer law. In this work, TTs were adjusted in the range from ca. $84 \%$ to $99 \%$.

On the basis of the results of De et al. [27], the above TT range is the percolation-type property of the SWCNT network film, rather than that of bulk-like. It is a characteristic that the percolation-type property is less dependent on the film thickness suggesting the formation of the twodimensional SWCNT network structure in this TT range. Typical AFM images of SWCNT network films with different
TTs are shown in Figure 4, which validate the formation of a two-dimensional network, even with a high transparency.

The bundle thickness was measured by analyzing the AFM images of SWCNT network films. The values of average bundle thickness are listed in Table 1. (The histograms of bundle thickness are shown in Figure S2 in Supplementary Materials.) The average bundle thickness is clearly larger than the above-discussed tube diameter: this suggests that the dilution process described in Experimental Methods causes slight flocculation of SWCNTs, but visible large SWCNT bundles are not formed in the diluted dispersion. In addition, such flocculation would induce the formation of the SWCNT bundles' network by van der Waals' interactions.

As shown in Table 1, the ultracentrifugation time dependence of the bundle thickness is extremely less. The average bundle thickness of UC10 is only $\sim 10 \%$ larger than that of UC60 with the same tube diameter. On the other hand, the average bundle thickness apparently shows tube diameter dependence; i.e., the smaller tube diameter leads to the smaller bundle thickness. By assuming that the SWCNT bundles show the close-packed structure of a triangular lattice model, the approximate number of SWCNTs in the bundle can be estimated. As shown in Figure 5, the number of SWCNTs in an average thickness bundle is approximately the same for each tube diameter.

Figures 6(a)-6(c) show AFM images of the SWCNT network films, in which SWCNTs form their random network structure. Although these films were prepared such that they exhibit approximately the same transparency $(\mathrm{TT}=\mathrm{ca} .94 \%)$, the network structure appears to become increasingly sparse 


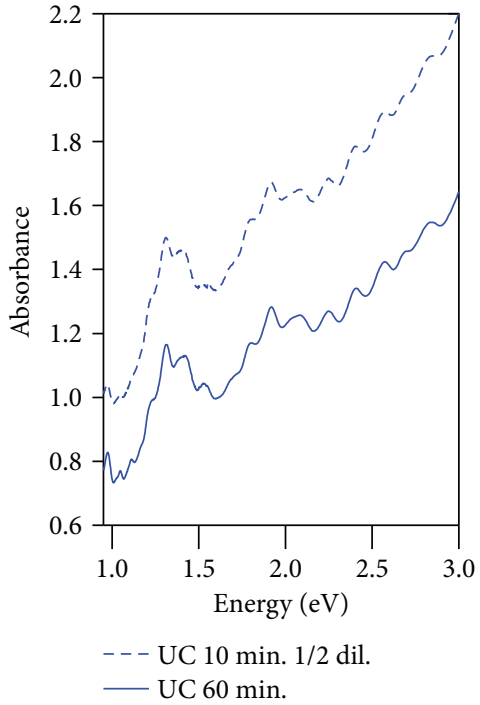

(a)

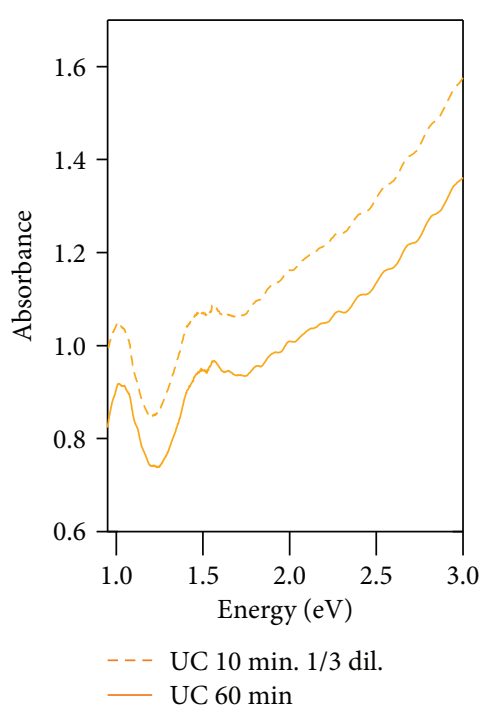

(b)

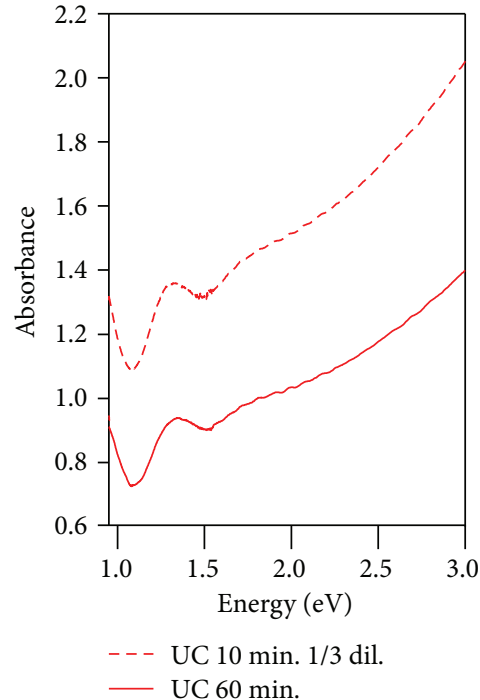

(c)

Figure 2: UV-vis-NIR spectra of UC10 and UC60 with different tube diameters of (a) $1.3 \mathrm{~nm}$, (b) $1.7 \mathrm{~nm}$, and (c) $2.0 \mathrm{~nm}$. Each figure indicates two samples prepared by different ultracentrifugation times of $10 \mathrm{~min}$ (dash lines) and $60 \mathrm{~min}$ (solid lines). The UC10 samples were 2-3 times diluted for the measurements as indicated.

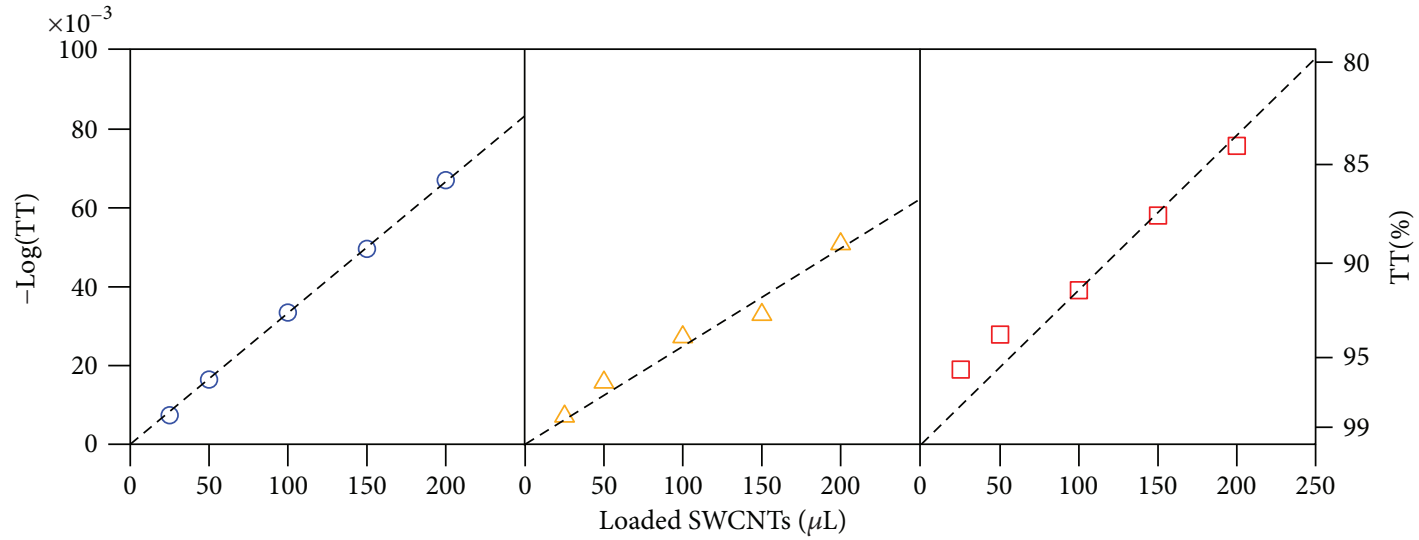

(a)

(b)

(c)

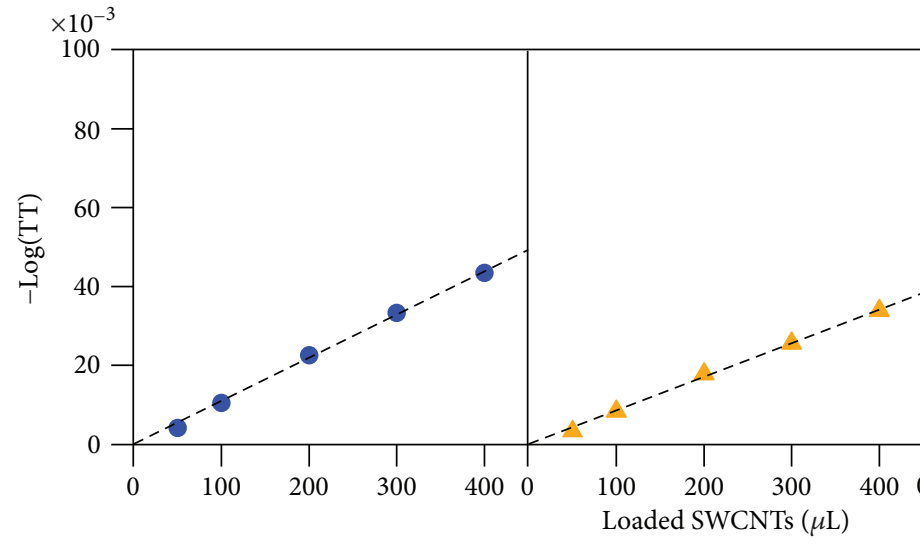

(d)

(e)

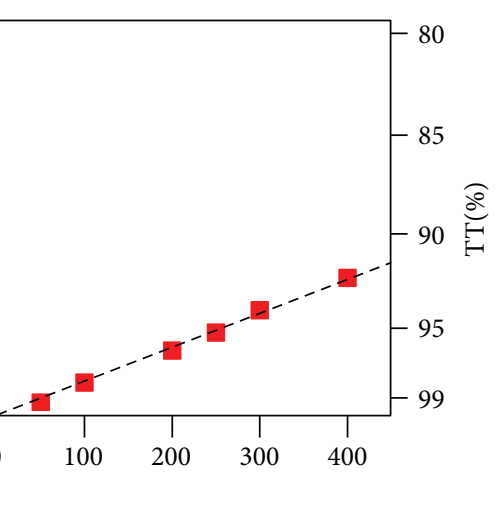

(f)

FIGURE 3: Relationship between the total light transmittance (TT) and the loaded amount of SWCNTs in the fabrication of SWCNT network films with different tube diameters of ( $a$ and d) $1.3 \mathrm{~nm}$, ( $b$ and e) $1.7 \mathrm{~nm}$, and ( $c$ and f) $2.0 \mathrm{~nm}$. Ultracentrifugation durations for the preparation of the dispersions are $10 \mathrm{~min}(\mathrm{a}-\mathrm{c})$ and $60 \mathrm{~min}(\mathrm{~d}-\mathrm{f})$. 


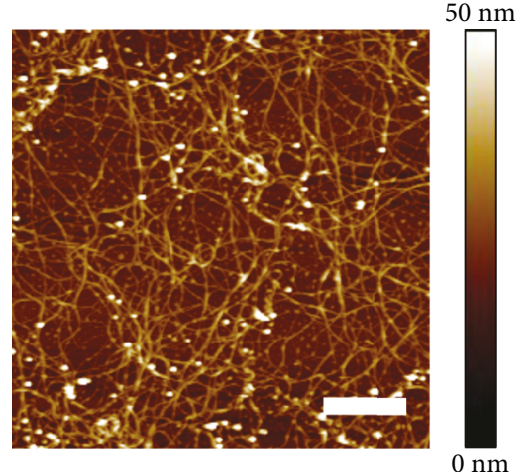

(a)

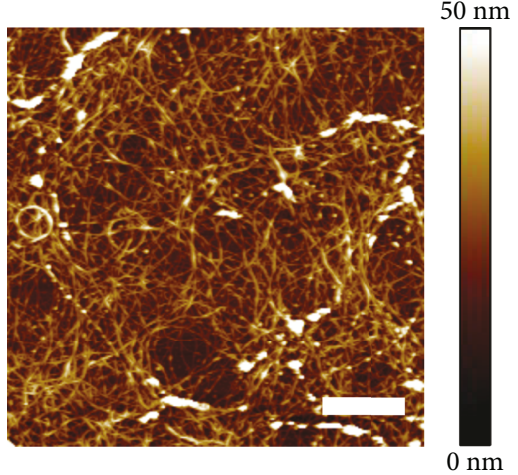

(b)

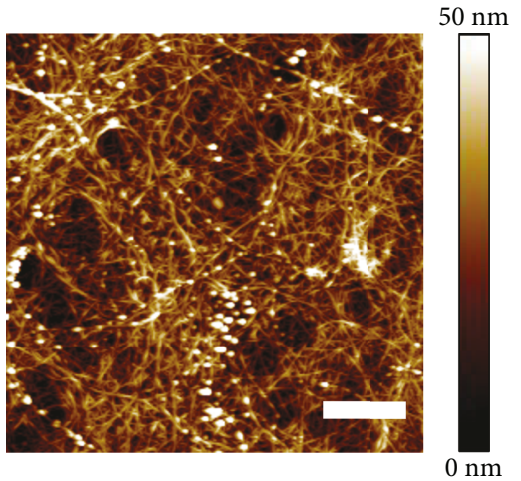

(c)

Figure 4: Typical AFM images of SWCNT TCFs with different SWCNT network densities (1.3 nm, UC10). Scale bars are $1 \mu \mathrm{m}$. Transmittances of (a) 98.3\%, (b) 92.6\%, and (c) $85.7 \%$ were measured in these SWCNT TCFs.

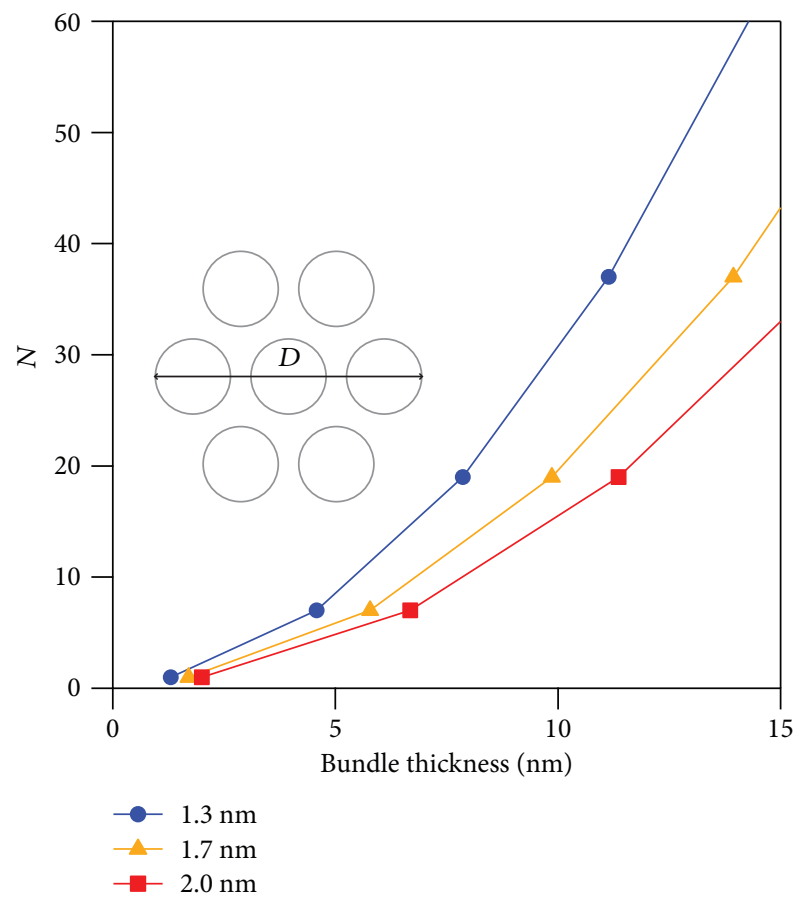

FIGURE 5: Relationship between the thickness of SWCNT bundles and the number of SWCNTs in an average thickness bundle $(N)$ for each tube diameter. The inset shows the close-packed model used in this calculation. $D$ and $a$ denote the SWCNT bundle thickness and SWCNT-SWCNT distance $(0.34 \mathrm{~nm})$, respectively.

with an increase in the tube diameter. Because the number of SWCNTs in an average thickness bundle is approximately the same irrespective of the tube diameter as discussed above, the optical extinction of the SWCNT bundle per unit length should increase with an increase in tube diameter. Therefore, the preparation of SWCNT network films with the same transparency by using SWCNT samples with different average tube diameters could cause a change in the density of the SWCNT network structure. Thus, the total length of the conduction pathways in the SWCNT network should decrease with an increase in the tube diameter. The difference in the network density observed in Figures 6(a)-6(c) strongly supports the abovementioned relationship between the tube diameter and the total length of conduction pathways.

3.3. Transparent Conductivity of the SWCNT Network Film and Percolation Analysis. In order to characterize the transparent conductivity performance of SWCNT network films, their sheet resistances $\left(R_{s}\right)$ were measured and plotted as a function of TT as shown in Figure 7(a). The relationship between TT and $R_{s}$ clearly indicates that a smaller tube diameter and a shorter centrifugation time result in superior transparent conductivity. Because the short ultracentrifugation time results in a long average length of SWCNTs as discussed above, the observed effect of the centrifugation time on the transparent conductivity is convincing. As for the tube diameter dependence observed on the transparent conductivity performance, it would be due to the change in the SWCNT network density caused by the difference in the tube diameter. Because SWCNTs with a small diameter tend to form a dense network (Figures 6(a)-6(c)), the number of conductive pathways in SWCNT network films increases with a decrease in tube diameter as discussed above. The dense SWCNT network structure with the high transparency should therefore lead to improve overall performance.

For further analysis of transparent conductivity, as shown in Figure $7(\mathrm{a})$, we performed data fitting by using the relationship between TT and $R_{s}$ in the percolation region defined by De et al. [27]. TT of SWCNT network films in the percolation region is expressed as follows:

$$
\mathrm{TT}=\left[1+\frac{1}{\prod}\left(\frac{Z_{0}}{R_{s}}\right)^{1 /(n+1)}\right]^{-2},
$$

where $\prod, Z_{0}, R_{s}$, and $n$ are the percolative figure of merit (FoM), the impedance of free space $(377 \Omega)$, sheet resistance, and the percolation exponent, respectively. Here, $\Pi$ is described as follows: 


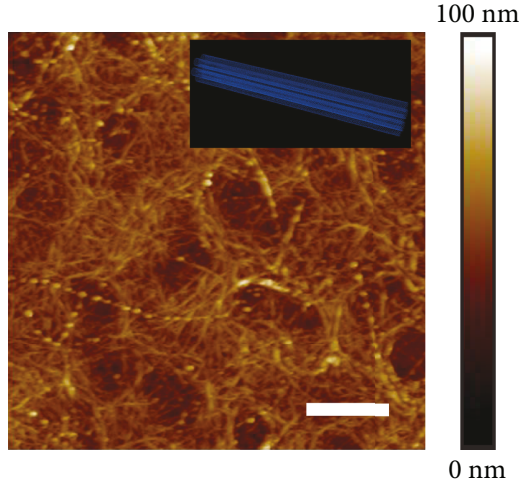

(a)

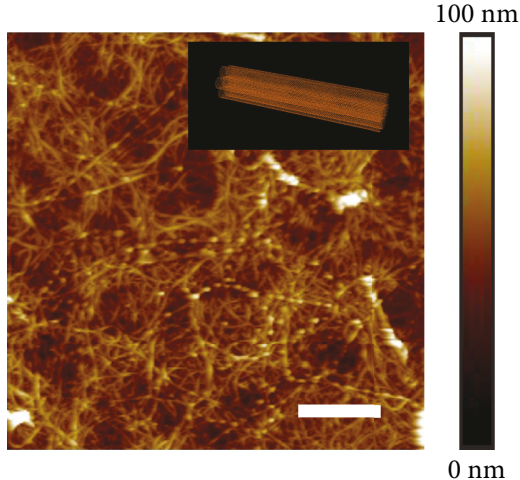

(b)

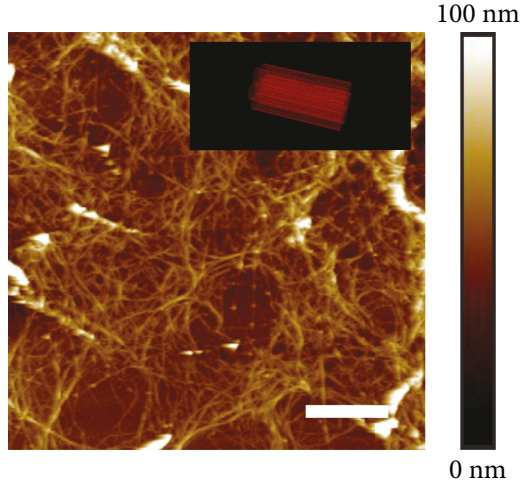

(c)

FIGURE 6: Typical AFM images of SWCNT network films that exhibit $~ 94 \%$ transmittance and that are prepared by using SWCNT samples with different tube diameters: (a) $1.3 \mathrm{~nm}$, (b) $1.7 \mathrm{~nm}$, and (c) $2.0 \mathrm{~nm}$. Centrifugation time is $60 \mathrm{~min}$. Scale bars are $1 \mu \mathrm{m}$.

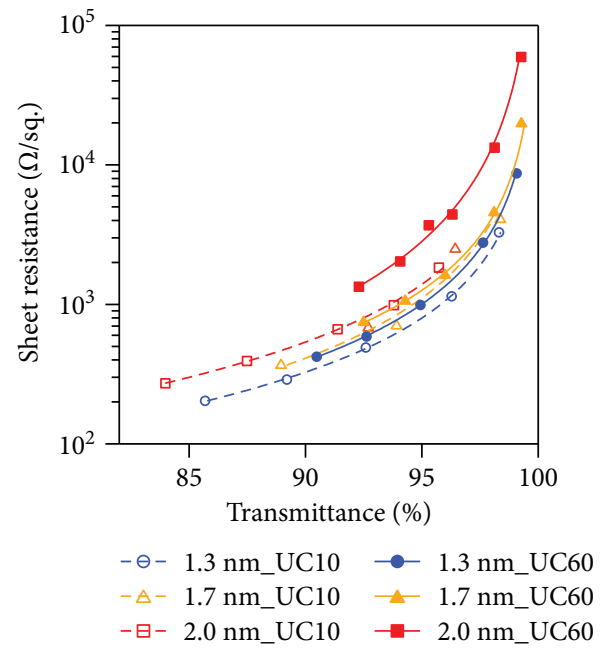

(a)

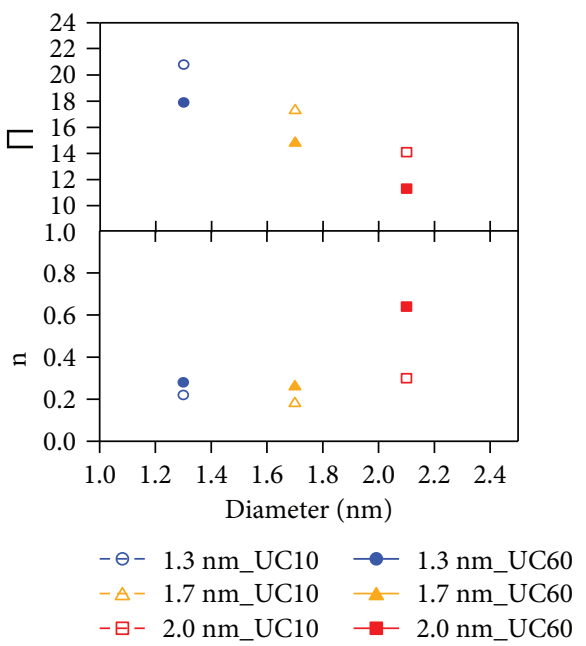

(b)

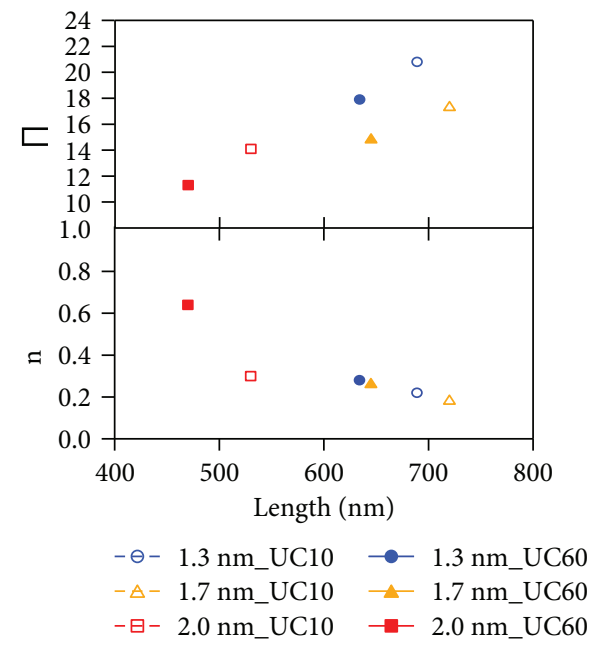

(c)

Figure 7: The performance of SWCNT TCFs. (a) Sheet resistance plotted as a function of total light transmittance in SWCNT TCFs fabricated by using various SWCNT dispersions of different tube diameters (blue: $1.3 \mathrm{~nm}$, orange: $1.7 \mathrm{~nm}$, red: $2.0 \mathrm{~nm}$ ). Solid and dashed lines are obtained by least square fitting of (1). (b and c) FoM ( $\left.\prod\right)$ and percolation exponent $(n)$ plotted as a function of the tube diameter (b) and the average tube length (c). 


$$
\Pi=2\left[\frac{\sigma_{\mathrm{DC}} / \sigma_{\mathrm{Op}}}{\left(Z_{0} t_{\min } \sigma_{\mathrm{Op}}\right)^{n}}\right]^{1 /(n+1)},
$$

where $\sigma_{\mathrm{DC}}, \sigma_{\mathrm{Op}}$, and $t_{\text {min }}$ are DC conductivity, optical conductivity, and critical film thickness below which DC conductivity shows the film thickness dependency, respectively.

FoM (П) can be considered to represent the transparent conductivity performance, while the percolation exponent (n) is related to the dimensions of the SWCNT network structure. As discussed above, the dimensions of the SWCNT network structure (and thus, the transparent conductivity) in the present TT range are only slightly dependent on the film thickness. Therefore, the percolation exponent $(n)$ is assumed to be the same for the SWCNT network films fabricated using the same dispersion. In other words, we have adopted $\prod$ and $n$ as the independent variables in the fitting of the experimental results by using (1).

As shown in Figure 7(a), the relationship between TT and $R_{s}$ closely follows (1). The values of $\prod$ and $n$ obtained by data fitting for each SWCNT network film are plotted against the tube diameter and the average tube length in Figures $7(\mathrm{~b})$ and $7(\mathrm{c})$, respectively. It was confirmed that $\prod$ ranging from 11.3 to 20.8 are typical for SWCNT network films [27], representing that the transparent conductivity performance of the SWCNT network film in the present study is comparable to those of the previous reports [27]. Furthermore, the abovementioned correlation between $\prod$ and the tube diameter is shown in Figure $7(b)$; i.e., the smaller the tube diameter is, the superior the transparent conductive performance is. On the other hand, these values of the percolation exponent $n$ ranging from 0.26 to 0.64 , as well as the reported ones [27], are considerably smaller than the universal value of the two-dimensional system $(n=1.3)$ [33], probably because the anisotropic structure of SWCNT bundles causes the interactions among them to be different from that in the case of the universal system [33].

The correlation between the percolation exponent $n$ and the tube length was observed in Figure $7(\mathrm{c})$. In addition, it was also observed that the percolation exponent of UC60 is slightly larger than that of UC10 for each tube diameter, as shown in Figure 7(b). These results strongly suggest that $n$ might be a reliable indicator of the tube length and the connectivity in the SWCNT network films. In general, the decrease in the percolation exponent indicates an improvement in the local connectivity and, thus, the electric conductivity of the network. Therefore, a possible explanation for the unidirectional dependence of $n$ on the ultracentrifugation time is that the shorter average length of SWCNTs in UC60 causes the sharper decrease in the electrical conductivity than UC10 as the SWCNT network density reduces toward the percolation threshold. Recently, it has been reported that the infrared (IR) absorption spectra originating from the intraband transition of SWCNTs reflect the length and conductivity of SWCNTs $[12,34]$. The comparison study between the percolation exponent $n$ and the behavior of IR absorption would be anticipated in future work to explore the detailed interpretation of $n$ in the transparent conductivity of SWCNT network films.

\section{Conclusions}

In this study, we have investigated the structural characteristics and the transparent conductivity properties of SWCNT network films prepared by the filtration and transfer method, by focusing on the relationship between the tube diameter and length of individual SWCNTs and their network structure. Furthermore, the figure of merit and the percolation exponent were analyzed based on data fitting of the transparent conductivity properties of SWCNT network films by using the percolation model. By analyzing the AFM images of SWCNT network films, it was found that a narrow tube diameter results in narrow bundles that lead to form their dense network. Such dense network of SWCNTs with narrow tube diameter improved the transparent conductivity property because of the increased total length of conduction pathways with a relatively high transparency. In addition, it was also confirmed that a shorter centrifugation time results in a longer average length of SWCNTs and, thus, superior transparent conductivity. The observed structural effects on the transparent conductivity of the SWCNT network film were consistent with the dependence of the obtained figure of merit and the percolation exponent on the tube diameter and length. Furthermore, it was suggested that the percolation exponent might be a reliable indicator of the tube length and the connectivity in the SWCNT network films. These results would be beneficial for determining the optimum structure of SWCNTs (as well as their secondary features such as the bundle dimensions and network connectivity) for TCF applications.

\section{Data Availability}

The data used to support the findings of this study are available from the corresponding author upon request.

\section{Conflicts of Interest}

The authors declare that they have no conflicts of interest.

\section{Acknowledgments}

This work has been supported by the New Energy and Industrial Technology Development Organization (NEDO).

\section{Supplementary Materials}

Figure S1: length distributions of SWCNTs with different tube diameters of ( $a$ and d) $1.3 \mathrm{~nm}$, (b and e) $1.7 \mathrm{~nm}$, and (c and f) $2.0 \mathrm{~nm}$ in their dispersions. Ultracentrifugation conditions for the preparation of these dispersions are $100,000 \times \mathrm{g}$ for $10 \mathrm{~min}(\mathrm{a}-\mathrm{c})$ and $60 \mathrm{~min}(\mathrm{~d}-\mathrm{f})$. Each inset shows the typical AFM images, and the scale bar is $2 \mu \mathrm{m}$. Figure S2: histogram of the bundle thickness in SWCNT network films: (a and d) $1.3 \mathrm{~nm}$, (b and e) $1.7 \mathrm{~nm}$, and (c and f) $2.0 \mathrm{~nm}$. Ultracentrifugation conditions are $100,000 \times \mathrm{g}$ for $10 \mathrm{~min}(\mathrm{a}-\mathrm{c})$ and $60 \mathrm{~min}(\mathrm{~d}-\mathrm{f})$. (Supplementary Materials) 


\section{References}

[1] Z. Wu, Z. Chen, X. Du et al., "Transparent, conductive carbon nanotube films," Science, vol. 305, no. 5688, pp. 1273-1276, 2004.

[2] Y. I. Song, C.-M. Yang, D. Y. Kim, H. Kanoh, and K. Kaneko, "Flexible transparent conducting single-wall carbon nanotube film with network bridging method," Journal of Colloid and Interface Science, vol. 318, no. 2, pp. 365-371, 2008.

[3] M. F. Islam, E. Rojas, D. M. Bergey, A. T. Johnson, and A. G. Yodh, "High weight fraction surfactant solubilization of single-wall carbon nanotubes in water," Nano Letters, vol. 3, no. 2, pp. 269-273, 2003.

[4] M. J. O'Connell, P. Boul, L. M. Ericson et al., "Reversible water-solubilization of single-walled carbon nanotubes by polymer wrapping," Chemical Physics Letters, vol. 342, no. 3-4, pp. 265-271, 2001.

[5] L. Hu, D. S. Hecht, and G. Grüner, "Carbon nanotube thin films: fabrication, properties, and applications," Chemical Reviews, vol. 110, no. 10, pp. 5790-5844, 2010.

[6] D. S. Hecht, L. Hu, and G. Irvin, "Emerging transparent electrodes based on thin films of carbon nanotubes, graphene, and metallic nanostructures," Advanced Materials, vol. 23, no. 13, pp. 1482-1513, 2011.

[7] H.-Z. Geng, K. K. Kim, K. P. So, Y. S. Lee, Y. Chang, and Y. H. Lee, "Effect of acid treatment on carbon nanotube-based flexible transparent conducting films," Journal of the American Chemical Society, vol. 129, no. 25, pp. 7758-7759, 2007.

[8] Y. Kim, M. Chikamatsu, R. Azumi, T. Saito, and N. Minami, "Industrially feasible approach to transparent, flexible, and conductive carbon nanotube films: cellulose-assisted film deposition followed by solution and photonic processing," Applied Physics Express, vol. 6, no. 2, article 025101, 2013.

[9] Y. Zhou, S. Shimada, T. Saito, and R. Azumi, "Building interconnects in carbon nanotube networks with metal halides for transparent electrodes," Carbon, vol. 87, pp. 61-69, 2015.

[10] Y. Kuwahara, F. Nihey, S. Ohmori, and T. Saito, "Length dependent performance of single-wall carbon nanotube thin film transistors," Carbon, vol. 91, pp. 370-377, 2015.

[11] Y. Asada, F. Nihey, S. Ohmori, H. Shinohara, and T. Saito, "Diameter-dependent performance of single-walled carbon nanotube thin-film transistors," Advanced Materials, vol. 23, no. 40, pp. 4631-4635, 2011.

[12] M. V. Shuba, A. G. Paddubskaya, A. O. Plyushch et al., "Experimental evidence of localized plasmon resonance in composite materials containing single-wall carbon nanotubes," Physical Review B, vol. 85, no. 16, article 165435, 2012.

[13] M. V. Shuba, A. G. Paddubskaya, P. P. Kuzhir et al., "Soft cutting of single-wall carbon nanotubes by low temperature ultrasonication in a mixture of sulfuric and nitric acids," Nanotechnology, vol. 23, no. 49, article 495714, 2012.

[14] G. E. Pike and C. H. Seager, "Percolation and conductivity: a computer study. I," Physical Review B, vol. 10, no. 4, pp. 1421$1434,1974$.

[15] J. Hicks, A. Behnam, and A. Ural, "Resistivity in percolation networks of one-dimensional elements with a length distribution," Physical Review E, vol. 79, no. 1, article 012102, 2009.

[16] R. M. Mutiso and K. I. Winey, "Electrical percolation in quasi-two-dimensional metal nanowire networks for transparent conductors," Physical Review E, vol. 88, no. 3, article 032134, 2013.
[17] D. Hecht, L. Hu, and G. Grüner, "Conductivity scaling with bundle length and diameter in single walled carbon nanotube networks," Applied Physics Letters, vol. 89, no. 13, article 133112, 2006.

[18] D. Simien, J. A. Fagan, W. Luo, J. F. Douglas, K. Migler, and J. Obrzut, "Influence of nanotube length on the optical and conductivity properties of thin single-wall carbon nanotube networks," ACS Nano, vol. 2, no. 9, pp. 1879-1884, 2008.

[19] A. Kaskela, A. G. Nasibulin, M. Y. Timmermans et al., "Aerosol-synthesized SWCNT networks with tunable conductivity and transparency by a dry transfer technique," Nano Letters, vol. 10, no. 11, pp. 4349-4355, 2010.

[20] A. A. Green and M. C. Hersam, "Colored semitransparent conductive coatings consisting of monodisperse metallic single-walled carbon nanotubes," Nano Letters, vol. 8, no. 5, pp. 1417-1422, 2008.

[21] Y. Maeda, K. Komoriya, K. Sode et al., "Preparation and characterization of transparent and conductive thin films of single-walled carbon nanotubes," Nanoscale, vol. 3, no. 4, pp. 1904-1909, 2011.

[22] Z. Li, H. R. Kandel, E. Dervishi et al., "Comparative study on different carbon nanotube materials in terms of transparent conductive coatings," Langmuir, vol. 24, no. 6, pp. 26552662, 2008.

[23] Q. Liu, T. Fujigaya, H.-M. Cheng, and N. Nakashima, "Free-standing highly conductive transparent ultrathin singlewalled carbon nanotube films," Journal of the American Chemical Society, vol. 132, no. 46, pp. 16581-16586, 2010.

[24] Z. Tang and P. Sheng, Nanoscale Phenomena: Basic Science to Device Applications, Springer, New York, NY, USA, 2008.

[25] R. Saito, M. Fujita, G. Dresselhaus, and M. S. Dresselhaus, "Electronic-structure of chiral graphene tubules," Applied Physics Letters, vol. 60, no. 18, pp. 2204-2206, 1992.

[26] K. Mustonen, T. Susi, A. Kaskela et al., "Influence of the diameter of single-walled carbon nanotube bundles on the optoelectronic performance of dry-deposited thin films," Beilstein Journal of Nanotechnology, vol. 3, pp. 692-702, 2012.

[27] S. De, P. J. King, P. E. Lyons, U. Khan, and J. N. Coleman, "Size effects and the problem with percolation in nanostructured transparent conductors," ACS Nano, vol. 4, no. 12, pp. 70647072, 2010.

[28] T. Saito, S. Ohshima, T. Okazaki, S. Ohmori, M. Yumura, and S. Iijima, "Selective diameter control of single-walled carbon nanotubes in the gas-phase synthesis," Journal of Nanoscience and Nanotechnology, vol. 8, no. 11, pp. 6153-6157, 2008.

[29] A. Jorio, R. Saito, J. H. Hafner et al., "Structural $(n, m)$ determination of isolated single-wall carbon nanotubes by resonant Raman scattering," Physical Review Letters, vol. 86, no. 6, pp. 1118-1121, 2001.

[30] T. Saito, S. Ohmori, B. Shukla, M. Yumura, and S. Iijima, "A novel method for characterizing the diameter of single-wall carbon nanotubes by optical absorption spectra," Applied Physics Express, vol. 2, no. 9, article 095006, 2009.

[31] H. Shirae, D. Y. Kim, K. Hasegawa, T. Takenobu, Y. Ohno, and S. Noda, "Overcoming the quality-quantity tradeoff in dispersion and printing of carbon nanotubes by a repetitive dispersion-extraction process," Carbon, vol. 91, pp. 20-29, 2015.

[32] A. J. Blanch, C. E. Lenehan, and J. S. Quinton, "Parametric analysis of sonication and centrifugation variables for dispersion of single walled carbon nanotubes in aqueous solutions 
of sodium dodecylbenzene sulfonate," Carbon, vol. 49, no. 15, pp. 5213-5228, 2011.

[33] G. Stauffer, Introduction to Percolation Theory, Taylor \& Francis, London, 1982.

[34] M. V. Shuba, A. G. Paddubskaya, P. P. Kuzhir et al., "Temperature induced modification of the mid-infrared response of single-walled carbon nanotubes," Journal of Applied Physics, vol. 119, no. 10, article 104303, 2016. 


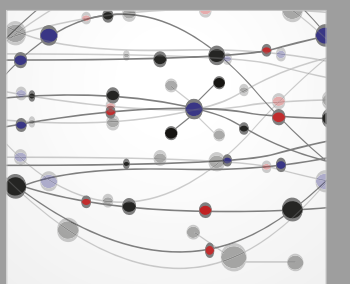

The Scientific World Journal
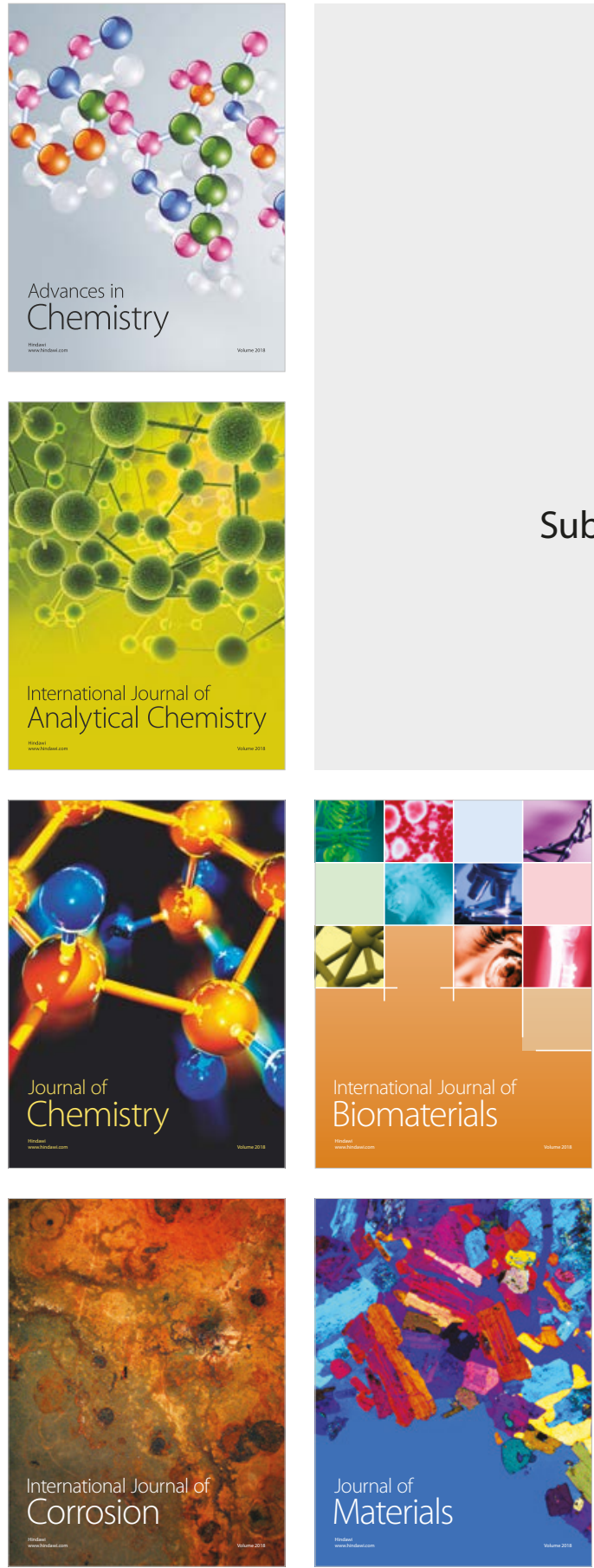

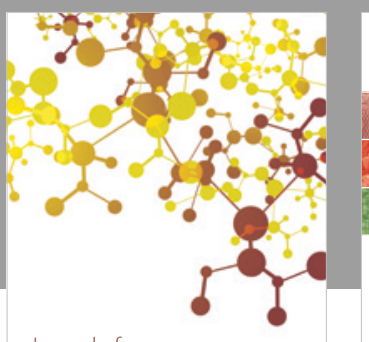

Journal of

Applied Chemistry
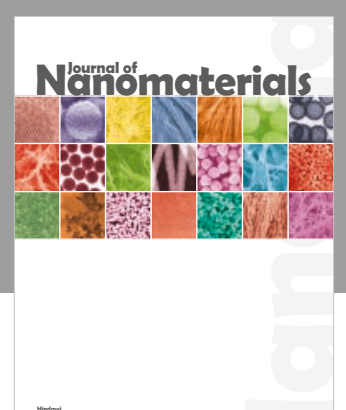

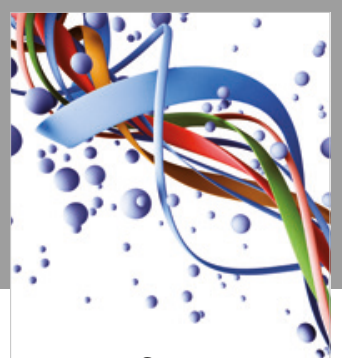

Scientifica

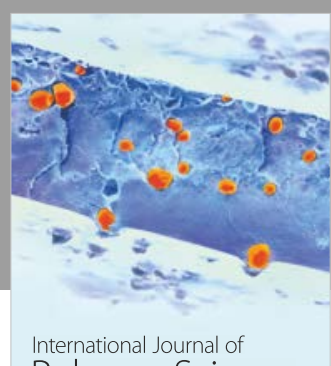

Polymer Science

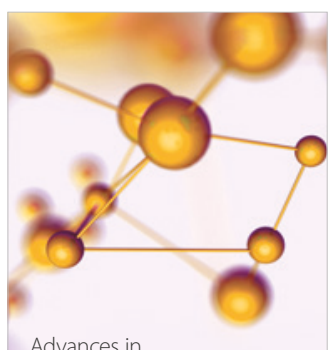

Physical Chemistry
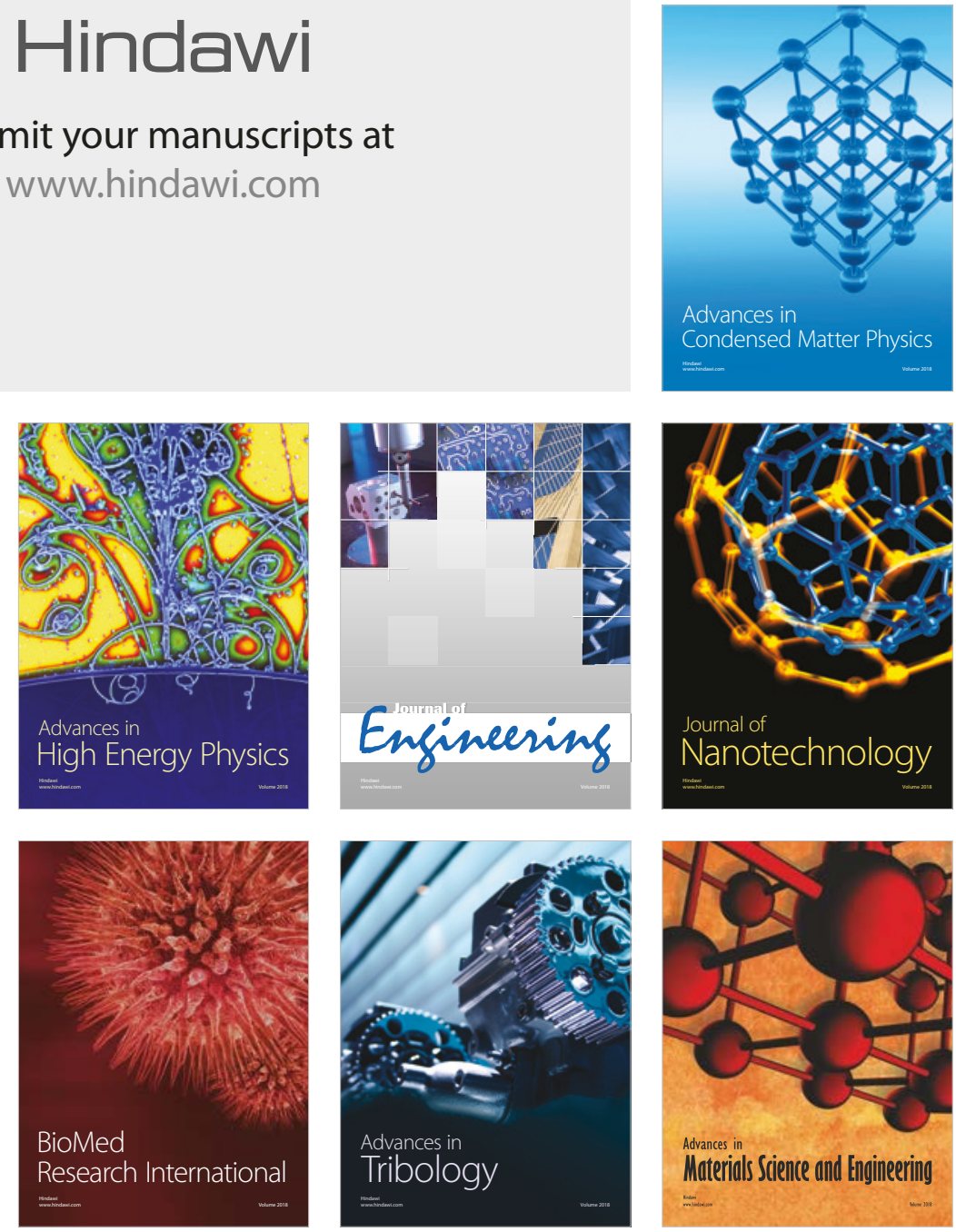